\title{
Bacteraemic pneumococcal pneumonia and SARS-CoV-2 pneumonia: differences and similarities
}

\author{
Leyre Serrano Fernández, MD ${ }^{\mathrm{a}, \mathrm{b}, *}$, Luis Alberto Ruiz Iturriaga, MD, PhD ${ }^{\mathrm{a}, \mathrm{b}}$, \\ Pedro Pablo España Yandiola, MD, PhD ${ }^{\mathrm{c}}$, Raúl Méndez Ocaña, MD ${ }^{\mathrm{d}, e}$, Silvia Pérez \\ Fernández ${ }^{\mathrm{f}}$, Eva Tabernero Huget, $\mathrm{MD}^{\mathrm{a}}$, Ane Uranga Echeverria, $\mathrm{MD}$, $\mathrm{PhD}^{\mathrm{c}}$, Paula Gonzalez \\ Jimenez, MD ${ }^{\text {d,e }}$, Patricia García Hontoria, MD ${ }^{c}$, Antoni Torres Martí, MD, PhD ${ }^{g}$, \\ Rosario Menendez Villanueva, MD, PhD ${ }^{\mathrm{d}, e, \dagger}$, Rafael Zalacain Jorge, MD, PhD ${ }^{\mathrm{a}, \dagger}$ \\ a Pneumology Service, Hospital Universitario Cruces, Barakaldo, Bizkaia, Spain \\ ${ }^{\mathrm{b}}$ Department of Immunology, Microbiology and Parasitology, Facultad de Medicina y Enfermería, Universidad del País Vasco/Euskal Herriko Unibertsitatea \\ UPV/EHU, Leioa, Bizkaia, Spain \\ ${ }^{\mathrm{c}}$ Pneumology Service, Hospital Universitario Galdakao-Usansolo, Galdakao, Bizkaia, Spain \\ d Pneumology Service, Hospital Universitari i politècnic La Fe, Valencia, Spain \\ e Instituto de Investigación Sanitaria La Fe, Valencia, Spain \\ ${ }^{\mathrm{f}}$ Bioinformatics and Statistics Unit, Biocruces Bizkaia Health Research Institute, Barakaldo. Bizkaia, Spain \\ g Pneumology Service, Hospital Clinic/Institut DÍnvestigacions Biomediques August Pi I Sunyer, University of Barcelona, Barcelona, Spain
}

\section{A R T I C L E I N F O}

\section{Article history:}

Received 25 October 2021

Revised 8 November 2021

Accepted 12 November 2021

\section{Keywords:}

Community-acquired pneumonia

Bacteraemic pneumococcal pneumonia

SARS-CoV-2

Process of care

Mortality

Survival

\begin{abstract}
A B S T R A C T
Objective: To analyse differences in clinical presentation and outcome between bacteraemic pneumococcal community-acquired pneumonia (B-PCAP) and sSvere Acute Respiratory Syndrome Coronavirus-2 (SARS-CoV-2) pneumonia.

Methods: This observational multi-centre study was conducted on patients hospitalized with B-PCAP between 2000 and 2020 and SARS-CoV-2 pneumonia in 2020. Thirty-day survival, predictors of mortality, and intensive care unit (ICU) admission were compared.

Results: In total, 663 patients with B-PCAP and 1561 patients with SARS-CoV-2 pneumonia were included in this study. Patients with B-PCAP had more severe disease, a higher ICU admission rate and more complications. Patients with SARS-CoV-2 pneumonia had higher in-hospital mortality ( $10.8 \%$ vs $6.8 \% ; P=0.004)$. Among patients admitted to the ICU, the need for invasive mechanical ventilation $(69.7 \%$ vs $36.2 \% ; P<0.001)$ and mortality were higher in patients with SARS-CoV-2 pneumonia. In patients with $\mathrm{B}-\mathrm{PCAP}$, the predictive model found associations between mortality and systemic complications (hyponatraemia, septic shock and neurological complications), lower respiratory reserve and tachypnoea; chest pain and purulent sputum were protective factors in these patients. In patients with SARS-CoV-2 pneu-
\end{abstract}

Abbreviations: AEMPS, Spanish Agency for Medicines and Health Products; B-PCAP, bacteraemic pneumococcal community-acquired pneumonia; CAP, community-acquired

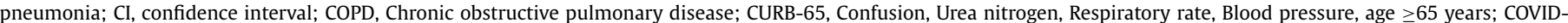

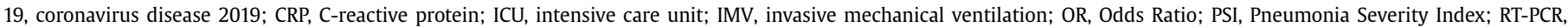
reverse transcription-polymerase chain reaction; SEPAR, Spanish Society of Pulmonology and Thoracic Surgery.

* Corresponding author at: Leyre Serrano Fernandez. Pneumology Service, Hospital Universitario Cruces, Plaza de Cruces s/n. 48903 Barakaldo, Bizkaia, Spain. Department

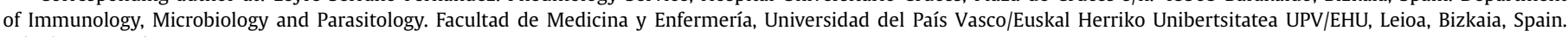
Telephone number: +34-94-6006510, Fax: +34-94-6006541.

E-mail addresses: leyre.serranofernandez@osakidetza.eus (L. Serrano Fernández), luisalberto.ruiziturriaga@osakidetza.eus (L.A. Ruiz Iturriaga),

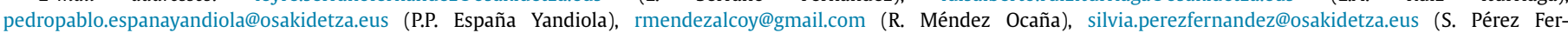

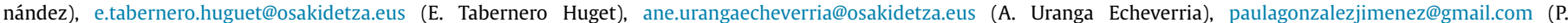

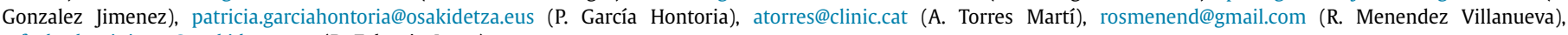
rafael.zalacainjorge@osakidetza.eus (R. Zalacain Jorge).

$\dagger$ These authors contributed equally as senior authors. 
monia, mortality was associated with previous liver and cardiac disease, advanced age, altered mental status, tachypnoea, hypoxaemia, bilateral involvement, pleural effusion, septic shock, neutrophilia and high blood urea nitrogen; in contrast, $\geq 7$ days of symptoms was a protective factor in these patients. In-hospital mortality occurred earlier in patients with B-PCAP.

Conclusions: Although B-PCAP was associated with more severe disease and a higher ICU admission rate, the mortality rate was higher for SARS-CoV-2 pneumonia and deaths occurred later. New prognostic scales and more effective treatments are needed for patients with SARS-CoV-2 pneumonia.

(c) 2021 The Author(s). Published by Elsevier Ltd on behalf of International Society for Infectious

Diseases.

This is an open access article under the CC BY-NC-ND license (http://creativecommons.org/licenses/by-nc-nd/4.0/)

\section{Background}

Streptococcus pneumoniae remains the most common cause of community-acquired pneumonia (Van der Poll and Opal, 2009; Johansson et al., 2010). Among pneumonia pathogens, it is the leading cause of hospitalization and death in adults (Roson et al., 2001; Shariatzadeh et al., 2005). Approximately 15-25\% of cases of pneumococcal pneumonia are bacteraemic (Said et al., 2013), and bacteraemic pneumococcal community-acquired pneumonia (B-PCAP) has traditionally been considered an invasive form of infection related to higher inflammatory status, worse in-hospital course and shorter long-term survival (Capelastegui et al., 2014; Ishiguro et al., 2016; Ruiz et al., 2019).

At the end of 2019, severe acute respiratory syndrome coronavirus-2 (SARS-CoV-2) emerged in China and rapidly developed into a global pandemic (Dhama et al., 2020; (Zhou et al., 2020a)., ). SARS-CoV-2 causes coronavirus disease 2019 (COVID19). The COVID-19 pandemic is a public health emergency, with more than 243 million confirmed cases and more than 4.9 million deaths.

Over recent months, numerous publications have sought to describe the stages of COVID-19; the clinical course, process of care, and predictive factors for poor outcome in SARS-CoV-2 pneumonia. However, few studies have compared the clinical features of SARS-CoV-2 pneumonia with other types of pneumonia (Tian et al., 2020; Zhao et al., 2020;(Zhou et al., 2020b) ; Shi et al., 2021).

The aim of the present study was to analyse the differences and similarities in clinical presentation, host inflammatory response and outcome between B-PCAP (which was previously the most common and invasive pneumonia) and SARS-CoV-2 pneumonia. Factors associated with intensive care unit (ICU) admission and inhospital mortality were compared, and survival after hospitalization was assessed for both types of pneumonia.

\section{Methods}

\section{Study design and population}

This observational multi-centre study was based on the analysis of a prospective registry of consecutive immunocompetent adults (aged $\geq 18$ years) hospitalized with B-PCAP between January 2000 and May 2020, and consecutive immunocompetent patients admitted with SARS-CoV-2 pneumonia between March and December 2020 (first and second waves of COVID-19 pandemic) to one of three tertiary medical centres (Cruces University Hospital, La Fe Hospital and Galdakao-Usansolo Hospital) in Spain. This study was approved by the corresponding ethics committee (Code PI2020083), and conducted in accordance with the principles of the Declaration of Helsinki on research in humans.

The bacteriological diagnosis of B-PCAP was based on a positive blood culture for $S$. pneumoniae taken within $24 \mathrm{~h}$ of presentation at hospital. Cases of SARS-CoV-2 pneumonia were confirmed by positive reverse transcription polymerase chain reaction (RTPCR) assay for the virus in nasopharyngeal swabs. Patients were excluded if they were known to be positive for human immunodeficiency virus or chronically immunosuppressed, or had been hospitalized for 14 days preceding the diagnosis of pneumonia.

\section{Study variables}

Patients' clinical and demographic characteristics were recorded, as well as physical examination, laboratory and radiological findings on admission. CURB-65 (confusion, urea nitrogen, respiratory rate, blood pressure, age $\geq 65$ years) (Lim et al., 2003) and Pneumonia Severity Index (PSI) (Fine et al., 1999) scores were used to assess the severity of pneumonia. Measures of in-hospital clinical course and outcome included: development of in-hospital complications; ICU admission; use of invasive mechanical ventilation (IMV); septic shock; in-hospital mortality; and length of hospital stay. Treatment of patients with COVID-19 was based on the recommendations issued by the Spanish Ministry of Health and the Spanish Agency for Medicines and Health Products (AEMPS) at the time of diagnosis (Documento técnico 19 March 2020)(http://www.aeemt.com/ web/wpcontent/uploads/2020/03/4_6026300193912129107.pdf, accessed April 2020;(AEMPS1) https://www.aemps.gob.es/laAEMPS/ docs/medicamentos-disponibles-SARS-CoV-2-19-3-2020.pdf, accessed May 2020;(AEMPS2) https://www.aemps.gob.es/la-aemps/ ultima-informacion-de-la-aemps-acerca-del-covid\%e2\%80\%

9119/tratamientos-disponibles-para-el-manejo-de-la-infeccion-

respiratoria-por-sars-cov-2/?lang=en, accessed September 2020;(COVID-19 Treatment Guidelines Panel 2020) https: //www.covid19treatmentguidelines.nih.gov/, accessed September 2020). Patients with B-PCAP were treated based on the guidelines of the Spanish Society of Pulmonology and Thoracic Surgery (Menéndez et al., 2010). In-hospital care and medical care following discharge were determined by patients' healthcare providers.

\section{Definitions}

Pneumonia was defined as the presence of new pulmonary infiltrates on chest X-ray, together with acute signs and symptoms suggestive of lower respiratory tract infection. The disease was classified as B-PCAP when the blood culture was positive for $S$. pneumoniae, and SARS-CoV-2 pneumonia when RT-PCR was positive for SARS-CoV-2 in nasopharyngeal swabs.

Patients were considered active smokers if they smoked at least 10 cigarettes per day, and heavy alcohol users if they reported a daily alcohol intake of at least $80 \mathrm{~g}$ for men or $60 \mathrm{~g}$ for women during the previous year (Grau et al., 2014). Septic shock was defined as systolic blood pressure $<90 \mathrm{mmHg}$ and the need for vasopressors for $\geq 4 \mathrm{~h}$ after fluid replacement therapy on admission ((Levy et al., 2003). 


\section{Statistical analysis}

Bivariate tables were constructed for patients with B-PCAP and SARS-CoV-2 pneumonia. Categorical variables were expressed as frequencies and percentages, and continuous variables were expressed as means (standard deviations) or medians (interquartile ranges) depending on whether or not data were normally distributed. For continuous variables, comparisons were performed using Student's $t$-test if the data followed a normal distribution, and using the Mann-Whitney $U$-test if the data did not follow a normal distribution. Chi-squared test or Fisher's exact test were used to compare qualitative variables.

Logistic regression models were performed to assess which variables were associated with ICU admission and use of IMV. Cox regression models were built to analyse in-hospital mortality. All variables with $P<0.100$ on bivariate analysis were included in logistic regression models. In the multi-variate logistic regression model, the variables with the highest $P$-values were eliminated one-by-one until all the variables entered were significant $(P<0.05)$. The results have been expressed as odd ratios (ORs) with corresponding $95 \%$ confidence intervals $(\mathrm{CI})$. In the case of patients with COVID-19, multiple imputations were used to impute any missing respiratory rate values.

Thirty-day survival was analysed using the Kaplan-Meier method. The log-rank test was used to compare survival between groups.

All analyses were performed using R Version 4.0.1.

\section{Results}

Six hundred and sixty-three patients hospitalized with B-PCAP and 1561 patients hospitalized with SARS-CoV-2 pneumonia were recruited into this study. Table 1 summarizes the patient baseline characteristics and in-hospital course overall and stratified by pneumonia aetiology. Patients with B-PCAP were older and more likely to have comorbidities. Focusing on clinical presentation, patients with B-PCAP were likely to have more severe disease, but the median duration of symptoms was shorter ( 3 vs 7 days; $P<0.001$ ). In addition, patients with B-PCAP had worse laboratory findings, including higher C-reactive protein level and lower lymphocyte count (median 710 vs $990 ; P<0.001$ ), but were less likely to have bilateral lung involvement on chest X-ray (16.9\% vs $71.8 \%$; $P<0.001$ ). Further, patients with B-PCAP were more frequently classified in the higher risk classes according to CURB-65 (2 to 5 ) and PSI $(4$ and 5$)$ scores $(P<0.001)$. Patients with B-PCAP were more frequently admitted to the ICU $(27.9 \%$ vs $12.9 \% ; P<0.001)$. In contrast, the IMV rate was similar in the two groups (10.1\% vs $9.1 \%$; $P=0.505)$. A higher percentage of patients with B-PCAP had complications during hospitalization. In contrast, patients with SARSCoV-2 pneumonia had a higher in-hospital mortality rate $(10.8 \%$ vs $6.8 \% ; P=0.004)$ and longer in-hospital stay.

Table 2 shows differences between patients admitted to the ICU with B-PCAP $(n=185)$ vs SARS-CoV-2 pneumonia $(n=201)$. The clinical presentation showed greater severity in patients with BPCAP, and these patients were in a higher risk class according to PSI and CURB-65 scores. In contrast, patients with SARS-CoV2 pneumonia were more likely to have bilateral lung involvement $(83.6 \%$ vs $34.6 \% ; P<0.001)$ and receive IMV (69.7\% vs $36.2 \%$; $P<0.001$ ). Thromboembolic complications, unlike all other complications, were more common in patients with SARS-CoV-2 pneumonia $(12.9 \%$ vs $2.2 \%)$. Patients with SARS-CoV-2 pneumonia admitted to the ICU, like the overall sample of patients with this type of pneumonia, had a higher in-hospital mortality rate $(23.9 \%$ vs $13 \%$; $P=0.009$ ) and longer in-hospital stay.

The results of multi-variate Cox regression of factors potentially associated with in-hospital mortality are listed in Table 3. In

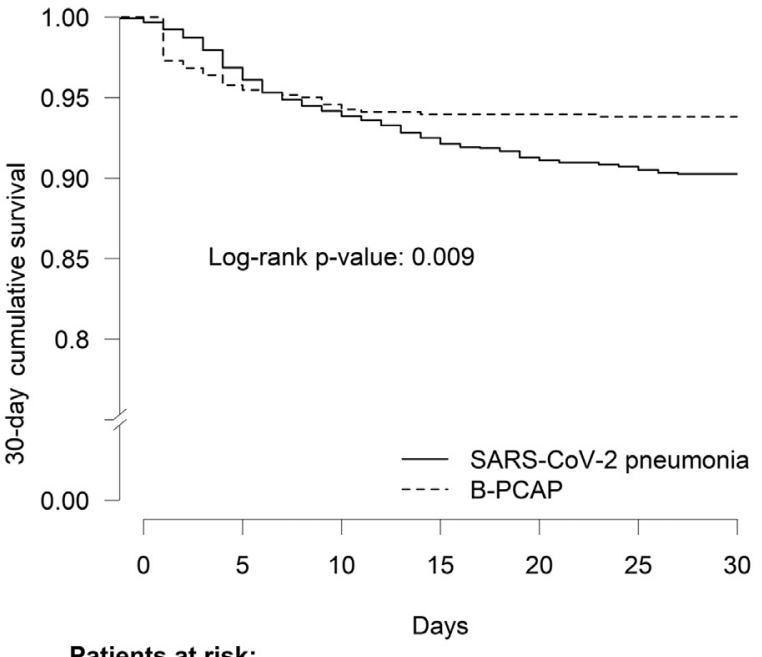

$\begin{array}{cccccccc}\text { SARS-CoV-2 } & 1560 & 1512 & 1470 & 1444 & 1425 & 1416 & 1409 \\ \text { B-PCAP } & 663 & 635 & 627 & 623 & 623 & 622 & 622\end{array}$

Figure 1. Thirty-day Kaplan-Meier survival curve for patients with bacteraemic pneumococcal community-acquired pneumonia (B-PCAP) and Severe Acute Respiratory Syndrome Coronavirus-2 (SARS-CoV-2) pneumonia.

the multi-variate Cox regression model, the following factors were independently associated with in-hospital mortality in patients with B-PCAP: chronic obstructive pulmonary disease (COPD) (OR 2.38, 95\% CI 1.19-4.78; $P=0.015)$; respiratory rate $\geq 30$ breaths $/ \mathrm{min}$ (OR 2.9, 95\% CI 1.43-5.89; $P=0.003$ ); sodium $<130 \mathrm{mmol} / \mathrm{L}(\mathrm{OR}$ 3.26, 95\% CI 1.37-7.81; $P=0.008$ ); neurological complications (OR 7.25, 95\% CI 3.52-14.93; $P<0.001$ ); and septic shock (OR 2.18, 95\% CI 1.06-4.48; $P=0.035$ ). Purulent sputum production (OR 0.4, 95\% CI $0.2-0.79 ; P=0.009$ ) and chest pain (OR 0.41 , 95\% CI $0.19-$ $0.88 ; P=0.022$ ) were protective factors. In patients with SARS-CoV2 pneumonia, predictors of in-hospital mortality were: age $\geq 65$ years (OR 4.777, 95\% CI 2.814-8.111; $P<0.001$ ); heart disease (OR 1.587, 95\% CI 1.007-2.341; $P=0.02$ ); liver disease (OR 2.365, 95\% CI 1.199-4.662); altered mental status (OR 2.464, 95\% CI 1.5863.827; $P<0.001$ ); respiratory rate $\geq 30$ breaths/min (OR $2.117,95 \%$ CI 1.362-3.289; $P<0.001$ ); hypoxaemia (OR 1.599 , 95\% CI 1.0452.447; $P=0.03$ ); blood urea nitrogen $\geq 30 \mathrm{mg} / \mathrm{dL}$ (OR $1.864,95 \% \mathrm{CI}$ 1.236-2.81; $P=0.003$ ); neutrophilia (each 10,000 -unit increase in neutrophil count $/ \mu \mathrm{L}$ ) (OR 1.052, 95\% CI 1.018-1.086; $P=0.002$ ); bilateral lung involvement (OR 1.868, 95\% CI 1.142-3.056; $P=0.013$ ); pleural effusion (OR 2.894, 95\% CI 1.282-6.536; $P=0.011$ ); and septic shock (OR 2.781, 95\% CI 1.703-4.542; $P<0.001$ ). Seven or more days of symptoms on admission was a protective factor against mortality (OR $0.464,95 \%$ CI $0.314-0.685 ; P<0.001$ ). The results of multi-variate logistic regression of factors associated with ICU admission and IMV in patients hospitalized with B-PCAP or SARSCoV-2 pneumonia are reported in Table 4 and Table S1 (see online supplementary material), respectively.

Figure 1 compares the 30-day Kaplan-Meier survival curves for patients with B-PCAP and SARS-CoV-2 pneumonia. The 30-day survival rate was significantly lower in patients with SARS-CoV-2 pneumonia (90.2\% vs $93.8 \%$; log rank $P \leq 0.009$ ).

\section{Discussion}

This study provides a comprehensive evaluation of host-related factors, process of care and outcome in a consecutive series of patients diagnosed with B-PCAP and SARS-CoV-2 pneumonia. The main findings were as follows: (1) patients with SARS-CoV-2 pneu- 
Table 1

General characteristics and in-hospital course overall and stratified by pneumonia aetiology.

\begin{tabular}{|c|c|c|c|c|}
\hline & All patients $(n=2224)$ & B-PCAP $(n=663)$ & $\begin{array}{l}\text { SARS-CoV-2 pneumonia } \\
(n=1561)\end{array}$ & $P$-value \\
\hline \multicolumn{5}{|l|}{ Demographic characteristics } \\
\hline Sex (male) & $1367(61.5 \%)$ & $427(64.4 \%)$ & $940(60.2 \%)$ & 0.071 \\
\hline Age $\geq 65$ years & $1005(45.1 \%)$ & $338(51 \%)$ & $667(42.7 \%)$ & 0.010 \\
\hline Active smoker & $293(13.3 \%)$ & $220(33.2 \%)$ & $73(4.8 \%)$ & $<0.001$ \\
\hline Alcoholism & $218(11.3 \%)$ & $115(17.6 \%)$ & $103(8.1 \%)$ & $<0.001$ \\
\hline Nursing home resident & $89(4 \%)$ & $18(2.7 \%)$ & $71(4.6 \%)$ & 0.057 \\
\hline \multicolumn{5}{|l|}{ Comorbid conditions } \\
\hline Comorbidities, yes & $1332(59.9 \%)$ & $394(59.4 \%)$ & $938(60.1 \%)$ & 0.807 \\
\hline Hypertension & $914(41.2 \%)$ & $280(42.5 \%)$ & $634(40.6 \%)$ & 0.440 \\
\hline Diabetes mellitus & $400(18 \%)$ & $109(16.4 \%)$ & $291(18.6 \%)$ & 0.240 \\
\hline Dyslipidaemia & $694(31.3 \%)$ & $156(23.7 \%)$ & $538(34.5 \%)$ & $<0.001$ \\
\hline Heart disease & $412(18.6 \%)$ & $173(26.3 \%)$ & $239(15.3 \%)$ & $<0.001$ \\
\hline Cerebrovascular disease & $92(4.8 \%)$ & $42(6.3 \%)$ & $50(3.9 \%)$ & 0.025 \\
\hline Chronic obstructive pulmonary disease & $218(9.8 \%)$ & $120(18.1 \%)$ & $98(6.3 \%)$ & $<0.001$ \\
\hline Liver disease & $90(4.1 \%)$ & $42(6.3 \%)$ & $48(3.1 \%)$ & 0.001 \\
\hline Chronic severe renal disease & $164(7.3 \%)$ & $45(6.8 \%)$ & $119(7.6 \%)$ & 0.548 \\
\hline Neoplastic disease & $143(6.4 \%)$ & $52(7.8 \%)$ & $91(5.8 \%)$ & 0.094 \\
\hline \multicolumn{5}{|l|}{ Clinical characteristics } \\
\hline Duration of symptoms (days), median (IQR) & $6(3-8)$ & $3(2-5)$ & $7(5-10)$ & $<0.001$ \\
\hline Altered mental status & $153(6.9 \%)$ & $70(10.6 \%)$ & $83(5.3 \%)$ & $<0.001$ \\
\hline Fever & $1649(74.5 \%)$ & $539(82.7 \%)$ & $1110(71.1 \%)$ & $<0.001$ \\
\hline Cough & $1627(73.6 \%)$ & $502(77 \%)$ & $1125(72.2 \%)$ & 0.022 \\
\hline Purulent sputum production & $361(16.3 \%)$ & $300(46 \%)$ & $61(3.9 \%)$ & $<0.001$ \\
\hline Dyspnoea & $1196(54 \%)$ & $384(58.9 \%)$ & $812(52 \%)$ & 0.004 \\
\hline Chest pain & $544(24.6 \%)$ & $338(51.8 \%)$ & $206(13.2 \%)$ & $<0.001$ \\
\hline \multicolumn{5}{|l|}{ Physical examination } \\
\hline Temperature $>39^{\circ} \mathrm{C}$ & $126(5.7 \%)$ & $83(12.5 \%)$ & $43(2.8 \%)$ & $<0.001$ \\
\hline Respiratory rate $\geq 30$ breaths $/ \mathrm{min}$ & $299(15.4 \%)$ & $172(26.4 \%)$ & $127(9.9 \%)$ & $<0.001$ \\
\hline Heart rate $>125$ beats $/ \mathrm{min}$ & $161(7.3 \%)$ & $115(17.3 \%)$ & $46(3 \%)$ & $<0.001$ \\
\hline Systolic blood pressure $<90 \mathrm{mmHg}$ & $83(3.8 \%)$ & $59(8.9 \%)$ & $24(1.6 \%)$ & $<0.001$ \\
\hline Oxygen saturation $<90 \%$ & $412(18.6 \%)$ & $216(33 \%)$ & $196(12.6 \%)$ & $<0.001$ \\
\hline \multicolumn{5}{|l|}{ Laboratory and radiological findings } \\
\hline Glucose, median (IQR), mg/dL & $114(100-142)$ & $129(107-166)$ & $110(99-130)$ & $<0.001$ \\
\hline Blood urea nitrogen $\geq 30 \mathrm{mg} / \mathrm{dl}$ & $492(22.2 \%)$ & $303(45.8 \%)$ & $189(12.1 \%)$ & $<0.001$ \\
\hline Sodium $<130 \mathrm{mmol} / \mathrm{L}$ & $83(3.7 \%)$ & $63(9.5 \%)$ & $20(1.3 \%)$ & $<0.001$ \\
\hline Haematocrit $<30 \%$ & $52(2.3 \%)$ & $19(2.9 \%)$ & $33(2.1 \%)$ & 0.361 \\
\hline C-reactive protein $\geq 150 \mathrm{mg} / \mathrm{L}$ & $449(22.7 \%)$ & $171(40.1 \%)$ & $279(17.9 \%)$ & $<0.001$ \\
\hline Platelet count $\times 10^{3} / \mu \mathrm{L}$, median (IQR) & $194(150-246)$ & $197(159-246)$ & $192(148-246)$ & 0.193 \\
\hline White blood cell count/ $\mu \mathrm{L}$, median (IQR) & $7090(5115-11408)$ & $\begin{array}{l}14,170 \\
(9600-20,100)\end{array}$ & $6170(4730-8070)$ & $<0.001$ \\
\hline 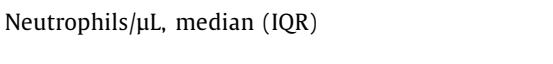 & $5400(3590-9514)$ & $\begin{array}{l}12,558 \\
(7900-17,676)\end{array}$ & $4500(3250-6240)$ & $<0.001$ \\
\hline Lymphocytes/ $\mu \mathrm{L}$, median (IQR) & $920(640-1299)$ & $710(417-1170)$ & $990(715-1330)$ & $<0.001$ \\
\hline Neutrophil/lymphocyte ratio, median (IQR) & $5.94(3.4-12.1)$ & $15.5(9.33-26.6)$ & $4.37(2.93-7.42)$ & $<0.001$ \\
\hline Lymphocyte/C-reactive protein ratio, median (IQR) & $14.4(5.82-36.2)$ & $8.05(2.43-24.6)$ & $15.9(7.08-42.5)$ & $<0.001$ \\
\hline \multicolumn{5}{|l|}{ Lung involvement on X-ray } \\
\hline Single lobe & $783(35.4 \%)$ & $424(64.7 \%)$ & $359(23.1 \%)$ & $<0.001$ \\
\hline Unilateral multi-lobe & $215(9.7 \%)$ & $120(18.3 \%)$ & $95(6.1 \%)$ & $<0.001$ \\
\hline Bilateral & $1228(55.5 \%)$ & $111(16.9 \%)$ & $1117(71.8 \%)$ & $<0.001$ \\
\hline Pleural effusion & $154(6.9 \%)$ & $119(17.9 \%)$ & $35(2.2 \%)$ & $<0.001$ \\
\hline \multicolumn{5}{|l|}{ Severity } \\
\hline Pneumonia Severity Index IV-V & $695(31.3 \%)$ & $364(55.5 \%)$ & $331(21.2 \%)$ & $<0.001$ \\
\hline CURB-65 $\geq 2$ & $774(35.2 \%)$ & $409(64.2 \%)$ & $365(23.4 \%)$ & $<0.001$ \\
\hline \multicolumn{5}{|l|}{ In-hospital course } \\
\hline Admission to ICU & $386(17.4 \%)$ & $185(27.9 \%)$ & $201(12.9 \%)$ & $<0.001$ \\
\hline Length of ICU stay (days), median (IQR) & $13(6-21)$ & $5(3-11)$ & $14(7-24)$ & $<0.001$ \\
\hline Need for mechanical ventilation & $209(9.4 \%)$ & $67(10.1 \%)$ & $142(9.1 \%)$ & 0.505 \\
\hline Length of IMV (days), median (IQR) & $14(8-22)$ & $10(4-18)$ & $13(8-21)$ & 0.563 \\
\hline Neurological complications & $94(4.2 \%)$ & $59(8.9 \%)$ & $35(2.2 \%)$ & $<0.001$ \\
\hline Renal complications & $259(11.7 \%)$ & $148(22.4 \%)$ & $111(7.1 \%)$ & $<0.001$ \\
\hline Cardiac complications & $212(9.7 \%)$ & $106(16.1 \%)$ & $106(6.9 \%)$ & $<0.001$ \\
\hline Thromboembolic complications & $55(2.8 \%)$ & $5(0.8 \%)$ & $50(3.9 \%)$ & $<0.001$ \\
\hline Haematological complications & $80(3.9 \%)$ & $58(10.1 \%)$ & $22(1.5 \%)$ & $<0.001$ \\
\hline Septic shock & $150(6.8 \%)$ & $116(17.7 \%)$ & $34(2.1 \%)$ & $<0.001$ \\
\hline \multicolumn{5}{|l|}{ Outcomes } \\
\hline In-hospital mortality & $214(9.6 \%)$ & $45(6.8 \%)$ & $169(10.8 \%)$ & 0.004 \\
\hline 30-day re-admission & $93(5.3 \%)$ & $18(2.7 \%)$ & $75(6.9 \%)$ & $<0.001$ \\
\hline Length of hospital stay (days), median (IQR) & $8(5-13)$ & $7(4-10)$ & $9(5-14)$ & $<0.001$ \\
\hline
\end{tabular}

B-PCAP, bacteraemic pneumococcal community-acquired pneumonia; SARS-CoV-2, severe acute respiratory syndrome coronavirus-2; ICU, intensive care unit; IMV, invasive mechanical ventilation; IQR, interquartile range. 
Table 2

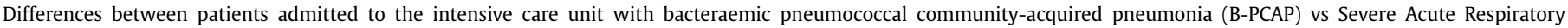
Syndrome Coronavirus-2 (SARS-CoV-2) pneumonia.

\begin{tabular}{|c|c|c|c|c|}
\hline & All patients $(n=386)$ & B-PCAP $(n=185)$ & $\begin{array}{l}\text { SARS-CoV-2 pneumonia } \\
(n=201)\end{array}$ & $P$-value \\
\hline \multicolumn{5}{|l|}{ Demographic characteristics } \\
\hline Sex (male) & $272(72.5 \%)$ & $131(70.8 \%)$ & $141(70.1 \%)$ & 0.976 \\
\hline Age $\geq 65$ years & $124(32.1 \%)$ & $68(36.8 \%)$ & $56(27.9 \%)$ & 0.854 \\
\hline Active smoker & $95(24.8 \%)$ & $87(47 \%)$ & $8(4 \%)$ & $<0.001$ \\
\hline Alcoholism & $69(20.1 \%)$ & $51(27.9 \%)$ & $18(11.2 \%)$ & $<0.001$ \\
\hline Nursing home resident & $6(1.5 \%)$ & $4(2.2 \%)$ & $2(1 \%)$ & 0.432 \\
\hline \multicolumn{5}{|l|}{ Comorbid conditions } \\
\hline Comorbidities, yes & $243(63 \%)$ & $101(54.6 \%)$ & $142(70.6 \%)$ & 0.002 \\
\hline Hypertension & $159(41.3 \%)$ & $64(34.8 \%)$ & $95(47.3 \%)$ & 0.017 \\
\hline Diabetes mellitus & $70(18.1 \%)$ & $22(11.9 \%)$ & $48(23.9 \%)$ & 0.003 \\
\hline Dyslipidaemia & $115(29.9 \%)$ & $42(22.8 \%)$ & $73(36.3 \%)$ & 0.005 \\
\hline Heart disease & $54(14 \%)$ & $27(14.7 \%)$ & $27(13.4 \%)$ & 0.839 \\
\hline Cerebrovascular disease & $10(2.9 \%)$ & $7(3.8 \%)$ & $3(1.9 \%)$ & 0350 \\
\hline Chronic obstructive pulmonary disease & $54(14 \%)$ & $39(21.1 \%)$ & $15(7.5 \%)$ & $<0.001$ \\
\hline Liver disease & $24(6.2 \%)$ & $20(10.8 \%)$ & $4(2 \%)$ & 0.001 \\
\hline Chronic severe renal disease & $21(5.4 \%)$ & $9(4.9 \%)$ & $12(5.9 \%)$ & 0.800 \\
\hline Neoplastic disease & $21(5.4 \%)$ & $10(5.4 \%)$ & $11(5.5 \%)$ & 1 \\
\hline \multicolumn{5}{|l|}{ Clinical characteristics } \\
\hline Duration of symptoms (days), median (IQR) & $5(3-7)$ & $3(2-5)$ & $7(5-9)$ & $<0.001$ \\
\hline Altered mental status & $38(9.8 \%)$ & $25(13.5 \%)$ & $13(6.5 \%)$ & 0.032 \\
\hline Fever & $292(76.2 \%)$ & $143(78.6 \%)$ & $149(74.1 \%)$ & 0.368 \\
\hline Cough & $293(76.5 \%)$ & $139(76.4 \%)$ & $154(76.6 \%)$ & 1 \\
\hline Purulent sputum production & $88(23 \%)$ & $82(45.1 \%)$ & $6(3 \%)$ & $<0.001$ \\
\hline Dyspnoea & $255(66.6 \%)$ & $124(68.1 \%)$ & $131(65.2 \%)$ & 0.614 \\
\hline Chest pain & $109(28.5 \%)$ & $92(50.5 \%)$ & $17(8.5 \%)$ & $<0.001$ \\
\hline \multicolumn{5}{|l|}{ Physical examination } \\
\hline Temperature $>39^{\circ} \mathrm{C}$ & $34(9.04 \%)$ & $24(13 \%)$ & $10(5.2 \%)$ & 0.015 \\
\hline Respiratory rate $\geq 30$ breaths/min & $143(40.6 \%)$ & $87(47.8 \%)$ & $56(32.9 \%)$ & 0.006 \\
\hline Heart rate $>125$ beats $/ \mathrm{min}$ & $63(16.3 \%)$ & $53(28.6 \%)$ & $10(5 \%)$ & $<0.001$ \\
\hline Systolic blood pressure $<90 \mathrm{mmHg}$ & $47(12.1 \%)$ & $44(23.8 \%)$ & $3(1.4 \%)$ & $<0.001$ \\
\hline Oxygen saturation $<90 \%$ & $155(40.2 \%)$ & $93(50.8 \%)$ & $62(30.8 \%)$ & 0.180 \\
\hline \multicolumn{5}{|l|}{ Laboratory and radiological findings } \\
\hline Glucose (mg/dL), median (IQR) & $120(102-150)$ & $120(99-153)$ & $121(106-147)$ & 0.363 \\
\hline Blood urea nitrogen $\geq 30 \mathrm{mg} / \mathrm{dL}$ & $139(36 \%)$ & $119(64.3 \%)$ & $20(9.9 \%)$ & $<0.001$ \\
\hline Sodium $<130 \mathrm{mmol} / \mathrm{L}$ & $34(8.8 \%)$ & $28(15.1 \%)$ & $6(2.9 \%)$ & 0.001 \\
\hline Haematocrit $<30 \%$ & $11(2.8 \%)$ & $5(2.7 \%)$ & $6(2.9 \%)$ & 0.544 \\
\hline C-reactive protein $\geq 150 \mathrm{mg} / \mathrm{L}$ & $108(33.5 \%)$ & $54(44.3 \%)$ & $54(27 \%)$ & 0.002 \\
\hline Platelet count $\times 10^{3} / \mu \mathrm{L}$, median (IQR) & $187(143-236)$ & $182(141-227)$ & $191(143-239)$ & 0.405 \\
\hline White blood cell count $/ \mu \mathrm{L}$, median (IQR) & $7750(5200-12100)$ & $\begin{array}{l}11300 \\
(6230-16300)\end{array}$ & $6595(5032-8418)$ & $<0.001$ \\
\hline 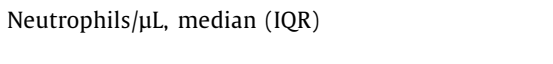 & $6420(3892-10902)$ & $\begin{array}{l}10081 \\
(5100-14626)\end{array}$ & $5105(3678-7192)$ & $<0.001$ \\
\hline Lymphocytes/ $\mu \mathrm{L}$, median (IQR) & $735(430-1082)$ & $564(300-1064)$ & $805(598-1090)$ & $<0.001$ \\
\hline Neutrophil/lymphocyte ratio, median (IQR) & $9.83(5.33-18.6)$ & $15.5(7.89-26.9)$ & $6.85(4.27-10.5)$ & $<0.001$ \\
\hline Lymphocyte/C-reactive protein ratio, median (IQR) & $7.5(2.9-17.4)$ & $4.59(1.62-15.7)$ & $9.4(4.36-17.6)$ & $<0.001$ \\
\hline \multicolumn{5}{|l|}{ Lung involvement on X-ray } \\
\hline \multicolumn{5}{|l|}{ Single lobe } \\
\hline Unilateral multi-lobe & $91(23.8 \%)$ & $63(34.6 \%)$ & $28(13.9 \%)$ & $<0.001$ \\
\hline \multirow[t]{2}{*}{ Bilateral } & $63(16.4 \%)$ & $56(30.8 \%)$ & $7(3.5 \%)$ & $<0.001$ \\
\hline & $231(60.3 \%)$ & $63(34.6 \%)$ & $168(83.6 \%)$ & $<0.001$ \\
\hline Pleural effusion & $41(10.6 \%)$ & $38(20.5 \%)$ & $3(1.5 \%)$ & $<0.001$ \\
\hline \multicolumn{5}{|l|}{ Severity } \\
\hline Pneumonia Severity Index IV-V & $186(48.3 \%)$ & $128(69.6 \%)$ & $58(28.9 \%)$ & $<0.001$ \\
\hline CURB-65 $\geq 2$ & $197(53.8 \%)$ & $137(75.7 \%)$ & $60(29.9 \%)$ & $<0.001$ \\
\hline \multicolumn{5}{|l|}{ In-hospital course } \\
\hline Need for mechanical ventilation & $207(53.6 \%)$ & $67(36.2 \%)$ & $140(69.7 \%)$ & $<0.001$ \\
\hline Length of IMV (days), median (IQR) & $13(7.25-21)$ & $10(4-18)$ & $13(8-21)$ & 0.563 \\
\hline \multicolumn{5}{|l|}{ Complications } \\
\hline Neurological complications & $48(12.5 \%)$ & $29(15.8 \%)$ & $19(9.6 \%)$ & 0.093 \\
\hline Stroke & $1(0.3 \%)$ & 0 & $1(0.7 \%)$ & 0.445 \\
\hline Renal complications & $128(33.4 \%)$ & $89(48.4 \%)$ & $39(19.6 \%)$ & $<0.001$ \\
\hline Cardiac complications & $90(23.8 \%)$ & $51(27.9 \%)$ & $39(20 \%)$ & 0.094 \\
\hline Thromboembolic complications & $26(7.3 \%)$ & $4(2.2 \%)$ & $22(12.9 \%)$ & $<0.001$ \\
\hline Haematological complications & $52(14.6 \%)$ & $46(27.9 \%)$ & $6(3.1 \%)$ & $<0.001$ \\
\hline Septic shock & $130(33.9 \%)$ & $107(57.8 \%)$ & $23(11.6 \%)$ & $<0.001$ \\
\hline \multicolumn{5}{|l|}{ Outcomes } \\
\hline In-hospital mortality & $72(18.7 \%)$ & $24(13 \%)$ & $48(23.9 \%)$ & 0.009 \\
\hline 30-day re-admission & $11(3.79 \%)$ & $5(2.7 \%)$ & $6(5.7 \%)$ & 0.218 \\
\hline Length of hospital stay, median (IQR) & $18(10-33)$ & $12(7-20)$ & $25(16-41)$ & $<0.001$ \\
\hline
\end{tabular}

IMV, invasive mechanical ventilation; IQR, interquartile range. 
Table 3

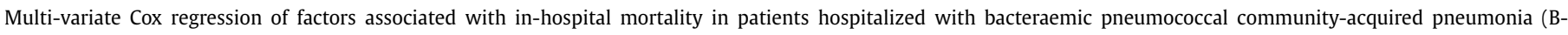
PCAP) and Severe Acute Respiratory Syndrome Coronavirus-2 (SARS-CoV-2) pneumonia.

\begin{tabular}{|c|c|c|c|c|c|}
\hline \multicolumn{3}{|l|}{ B-PCAP } & \multicolumn{3}{|l|}{ SARS-CoV-2 pneumonia } \\
\hline & OR $(95 \% \mathrm{CI})$ & $P$-value & & OR $(95 \% \mathrm{CI})$ & $P$-value \\
\hline Chronic obstructive pulmonary disease & $2.38(1.19-4.78)$ & 0.015 & Age $\geq 65$ years & $4.777(2.814-8.111)$ & $<0.001$ \\
\hline Purulent sputum production & $0.4(0.2-0.79)$ & 0.009 & Heart disease & $1.587(1.077-2.341)$ & 0.02 \\
\hline Chest pain & $0.41(0.19-0.88)$ & 0.022 & Liver disease & $2.365(1.199-4.662)$ & 0.013 \\
\hline Respiratory rate $\geq 30$ breaths $/ \mathrm{min}$ & $2.9(1.43-5.89)$ & 0.003 & Altered mental status & $2.464(1.586-3.827)$ & $<0.001$ \\
\hline Sodium $<130 \mathrm{mmol} / \mathrm{L}$ & $3.26(1.37-7.81)$ & 0.008 & $\geq 7$ days of symptoms & $0.464(0.314-0.685)$ & $<0.001$ \\
\hline Neurological complications ${ }^{\mathrm{a}}$ & $7.25(3.52-14.93)$ & $<0.001$ & Respiratory rate $\geq 30$ breaths/min & $2.117(1.362-3.289)$ & $<0.001$ \\
\hline \multirow[t]{6}{*}{ Septic shock } & $2.18(1.06-4.48)$ & 0.035 & Oxygen saturation $<90 \%$ & $1.599(1.045-2.477)$ & 0.03 \\
\hline & & & Blood urea nitrogen $\geq 30 \mathrm{mg} / \mathrm{dL}$ & $1.864(1.236-2.81)$ & 0.003 \\
\hline & & & Neutrophil count $/ \mu \mathrm{L} / 10,000^{\mathrm{b}}$ & $1.052(1.018-1.086)$ & 0.002 \\
\hline & & & Bilateral lung involvement & $1.868(1.142-3.056)$ & 0.013 \\
\hline & & & Pleural effusion & $2.894(1.282-6.536)$ & 0.011 \\
\hline & & & Septic shock & $2.781(1.703-4.542)$ & $<0.001$ \\
\hline
\end{tabular}

OR, odds ratio; $\mathrm{CI}$, confidence interval.

a Neurological complications: stroke, delirium and/or seizure.

b Neutrophil count is presented for each 10,000-unit increase.

Table 4

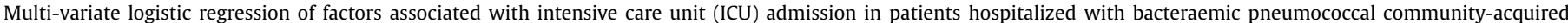
pneumonia (B-PCAP) and Severe Acute Respiratory Syndrome Coronavirus-2 (SARS-CoV-2) pneumonia.

\begin{tabular}{|c|c|c|c|c|c|}
\hline \multicolumn{3}{|l|}{ B-PCAP } & \multicolumn{3}{|l|}{ SARS-CoV-2 pneumonia } \\
\hline & OR $(95 \% \mathrm{CI})$ & $P$-value & & OR $(95 \% \mathrm{CI})$ & $P$-value \\
\hline Age $\geq 65$ years $^{\mathrm{a}}$ & $0.28(0.17-0.47)$ & $<0.001$ & Nursing home resident & $0.132(0.002-0.486)$ & 0.009 \\
\hline Active smoker & $2.09(1.29-3.4)$ & 0.003 & Age $\geq 65$ years $^{a}$ & $0.563(0.375-0.833)$ & 0.005 \\
\hline Respiratory rate $\geq 30$ breaths $/ \mathrm{min}$ & $3.39(2.11-5.46)$ & $<0.001$ & Respiratory rate $\geq 30$ breaths/min & $3.09(1.891-5)$ & $<0.001$ \\
\hline Systolic blood pressure $<90 \mathrm{mmHg}$ & $12.53(6.09-27.2)$ & $<0.001$ & Oxygen saturation $<90 \%$ & $4.483(2.812-7.125)$ & $<0.001$ \\
\hline Oxygen saturation $<90 \%$ & $2.39(1.46-3.92)$ & $<0.001$ & Lymphocytes $/ \mu \mathrm{L} / 1000^{c}$ & $0.364(0.231-0.557)$ & $<0.001$ \\
\hline Blood urea nitrogen $\geq 30 \mathrm{mg} / \mathrm{dL}$ & $3.45(2.15-5.63)$ & $<0.001$ & & & \\
\hline White blood cell count $/ \mu \mathrm{L} / 1000^{\mathrm{b}}$ & $0.95(0.92-0.98)$ & $<0.001$ & & & \\
\hline Bilateral lung involvement & $3.17(1.84-5.48)$ & $<0.001$ & & & \\
\hline
\end{tabular}

OR, odds ratio; $\mathrm{CI}$, confidence interval.

a Indicates the negative influence of advanced age on eligibility for ICU admission.

b White blood cell count is presented for each 1000-unit increase.

c Lymphocytes are presented for each 1000-unit increase.

monia had higher in-hospital mortality, although patients with BPCAP had more comorbidities, more severe clinical presentation and laboratory findings, higher ICU admission rates and in-hospital complications; (2) in comparison with patients with B-PCAP, patients with SARS-CoV-2 pneumonia requiring ICU admission were more likely to receive IMV and had poorer outcomes with a higher mortality rate; (3) factors and clinical scales (PSI and CURB-65) classically associated with severity and mortality on admission in patients with B-PCAP demonstrated lack of predictive value in patients with SARS-CoV-2 pneumonia; and (4) B-PCAP was characterized by early in-hospital mortality, whereas in-hospital mortality occurred later in patients with SARS-CoV-2 pneumonia.

The study sample is particularly interesting for several reasons: the types of pneumonia studied, the type of population studied, the size of the sample, and the multi-centre design of the study. The types of pneumonia that were compared were the pneumonia that has previously been the most common, invasive and virulent pneumonia, able to produce documented inflammatory status and systemic complications (B-PCAP, all cases diagnosed by positive blood culture), and the pneumonia caused by the current pandemic (SARS-CoV-2 pneumonia, all cases diagnosed by positive RT-PCR), which has also been related to exaggerated systemic inflammation. In terms of the type of population studied, only immunocompetent patients were included for both types of pneumonia. This enabled the authors to avoid the confounding effect of immunosuppression. Regarding the size of the sample and the multi-centre design of the study, to the authors' knowledge, this is among the largest series published on this topic including data for both types of pneumonia collected prospectively.
The results of this study illustrate that there are clear differences between the clinical presentation of B-PCAP and SARS-CoV2 pneumonia. B-PCAP can be considered a clear example of classic pneumonia, in which patients present with a history of a few days of typical symptoms, showing high severity on physical examination and laboratory parameters, and unilobar involvement on $\mathrm{X}$-ray. In contrast, SARS-CoV-2 pneumonia was observed to have a more dormant clinical course, bilateral lung involvement and higher in-hospital mortality. Few studies have compared COVID-19 with other types of pneumonia, and all previous series have compared SARS-CoV-2-positive pneumonia with SARS-CoV-2-negative pneumonia, without specifying the aetiology in the latter case (Tian et al., 2020; Zhao et al., 2020; (Zhou et al., 2020b)). Furthermore, previous studies included few cases, and just compared the groups in terms of general characteristics and in-hospital course. No previous studies performed and compared multi-variate analysis of severity or survival curves.

The present study confirmed the underestimation of the risk of death from viral pneumonia obtained with CURB-65 and PSI scores reported by previous studies (Guo et al., 2019; Nguyen et al., 2020). In the present series, these severity scales were not useful for identifying patients with severe SARS-CoV-2 pneumonia, as among patients with this type of pneumonia, just $28.9 \%$ of those admitted to the ICU had high-risk PSI scores and 29.9\% had high-risk CURB-65 scores, but $23.9 \%$ died during hospital admission. Moreover, in the entire sample of patients with SARS-CoV-2 pneumonia, only $21.2 \%$ and $23.4 \%$ were identified as high risk by the PSI score and the CURB-65 score, respectively, and the in-hospital mortality rate was $10.8 \%$. Wynants et al. (2020) published a systematic review of pre- 
diction models for COVID-19, and concluded that they were unable to recommend any of them for use in clinical practice.

The present study found that patients with B-PCAP had worse clinical presentation and laboratory findings, but in-hospital mortality was much higher in patients with SARS-CoV-2 pneumonia. The prognosis of B-PCAP has improved in recent years due to new vaccines (Whitney et al., 2003), early diagnosis and improvements in treatment (Gattarello et al., 2014). In contrast, SARS-CoV-2 pneumonia is a new illness and there is currently no specific antiviral treatment. Furthermore, although vaccines against COVID-19 are being administered in most countries, herd immunity has not yet been achieved. Moreover, the effectiveness of the vaccines is not the same in all patients, and the duration of immunity is not well known (Altawalah, 2021). The inflammatory response also plays an important role in COVID-19-related mortality. Previous research has suggested that excessive immune response triggers pathogenesis in other severe viral types of pneumonia, such as influenza and SARS (Van den Brand et al., 2014). It has also been documented that SARS-CoV-2 pneumonia triggers a strong innate inflammatory immune response which leads to a cytokine storm that causes acute respiratory distress syndrome (Xu et al., 2020; Ye et al., 2020).The present study did not find an association between in-hospital mortality in patients with COVID-19 and lymphopenia, whereas Zheng et al. (2020) did find an association in a meta-analysis analysing risk factors of critical cases of COVID19. This is despite the fact that the lymphocyte count on admission in the patients with SARS-CoV-2 pneumonia in the present study was low and similar to that documented by previous studies (Richardson et al., 2020), although curiously, the median lymphocyte count was lower among patients with B-PCAP than among patients with SARS-CoV-2 pneumonia in the present study. These results indicate that lymphopenia is associated with severity in any type of pneumonia, and is not specific for SARS-CoV-2 as has been reported previously (Méndez et al., 2019).

The present study found two distinct mortality mechanisms in the two types of pneumonia. In B-PCAP, the predictive model related mortality to systemic complications (hyponatraemia, septic shock and neurological complications), lower respiratory reserve (COPD) and high respiratory rate; these risk factors have been associated with mortality previously (Naucler et al., 2013) or are currently included in pneumonia severity scores (Fine et al., 1999; Lim et al., 2003). Chest pain and purulent sputum production were found to be protective factors against mortality in patients with BPCAP, probably because they were alarm symptoms that prompted the patient to seek medical attention and start treatment earlier (Fine et al., 1996). In contrast, in patients with SARS-CoV-2 pneumonia, mortality was more closely related to an exaggerated inflammatory response (blood urea nitrogen $\geq 30 \mathrm{mg} / \mathrm{dL}$ and neutrophilia) or respiratory failure (respiratory rate $\geq 30$ breaths/min, oxygen saturation $<90 \%$ and bilateral lung involvement). As in previous studies (Berenguer et al., 2020; Gallo et al., 2021), advanced age, altered mental status, and heart and liver diseases were associated with in-hospital mortality, and indicated worse baseline status. Further, septic shock was predictive of mortality, likely because it was related to bacterial superinfection, although microbiological aetiology data were not available. Pleural effusion was also related to in-hospital mortality, as this indicates severe inflammation and could be associated with viral pleuritis, bacterial superinfection or congestive heart failure (Zhan et al., 2021). Seven or more days of symptoms at hospital admission was found to be a protective factor against mortality for patients with SARS-CoV-2 pneumonia, as has been reported previously (Ciceri et al., 2020), probably because the patient is admitted at a later stage of the disease in which the risk of worsening is lower, or because the disease is milder and it takes longer to show severe symptoms.
Significant differences in the timing of in-hospital death were found between the groups. In patients with B-PCAP, in-hospital death occurred in the first few days after admission. As has been observed previously, pneumococcal mortality tends to be observed early, as prompt adequate targeted treatment improves outcomes in these patients (Garnacho-Montero et al., 2010). In contrast, in patients with SARS-CoV-2 pneumonia, survival started to decrease from day 4-5 of admission, this corresponding to day 10-12 after symptom onset, when the exaggerated inflammatory phase started (Siddiqi and Mehra, 2020).

Finally, regarding patients admitted to the ICU, the admission rate was lower in patients with SARS-CoV-2 pneumonia, but they had a longer ICU stay and greater need for IMV. These data reinforce the association of COVID-19 severity with respiratory failure, while the severity of B-PCAP was also associated with septic shock or other complications. All complications were observed more frequently in patients with B-PCAP, except for thromboembolic complications, which were more common in patients with SARS-CoV-2 pneumonia. Coagulopathy has been documented previously in patients with severe COVID-19, and this could lead to thromboembolic complications (Levi et al., 2020). Predictive factors associated with ICU admission in patients with B-PCAP were factors previously associated with severity (smoking, tachypnoea, hypotension, high blood urea nitrogen levels, respiratory failure or bilateral lung involvement). Older age had a negative association with ICU admission: as previous studies have documented, this indicates the negative influence of advanced age on eligibility for ICU admission (Boumendil et al., 2012; Ruiz et al., 2017). Leucocytosis was identified as protective against ICU admission, whereas leukopenia indicates abnormalities in the host's inflammatory response associated with increased susceptibility to severe disease and mortality (Hanada et al., 2016). Once again, in patients with SARS-CoV-2 pneumonia, respiratory failure (tachypnoea and oxygen saturation $<90 \%$ ) and cytokine storm (lymphopenia) were related to severity and ICU admission. Old age and nursing home residence were negatively associated with ICU admission; as in patients with B-PCAP, such patients were likely not eligible for ICU admission because of their poor baseline status.

This study has some limitations. It was an observational study and data on the two types of pneumonia were collected prospectively in different time periods. B-PCAP cases registered over 20 years were compared with SARS-CoV-2 cases registered over 10 months (March-December 2020), and this could introduce differences related to external uncontrollable factors. On the other hand, only patients from the first and second waves of the COVID19 pandemic were included. This may be a limitation but also a strength, because it enabled the authors to eliminate the bias of the pandemic effect that would have had to analyse the first-wave patients alone, and avoided the effect of vaccination on subsequent waves (in Spain, vaccination started in January 2021). Further, in patients with B-PCAP, data were not gathered on factors that seem to be commonly altered in SARS-CoV-2 pneumonia (i.e. D-dimer, lactate dehydrogenase or ferritin), so it was not possible to compare these laboratory data. Furthermore, some therapies such as high-flow oxygen therapy and extracorporeal membrane oxygenation have started to be used in recent years, and it was not possible to compare their effect in the two groups. On the other hand, as there have been millions of cases of SARS-CoV-2 pneumonia in a short time, the authors consider that the approach followed is the only way to compare patients with this type of pneumonia with a similarly sized sample of patients with B-PCAP.

\section{Conclusions}

This study examined differences between general characteristics, clinical presentation and in-hospital course of patients with B- 
PCAP and SARS-CoV-2 pneumonia, and analysed factors associated with in-hospital mortality and ICU admission in both groups. This study demonstrated differences in the behaviour of the two entities, which may facilitate differential diagnosis and enable the provision of differentiated treatment. Although patients with B-PCAP have more severe disease on admission and a higher ICU admission rate, in-hospital mortality associated with SARS-CoV-2 pneumonia is higher and occurs later during hospital admission. These results reinforce the need for new prognostic scales and effective treatment for patients with SARS-CoV-2 pneumonia.

\section{Declaration of Competing Interest}

None declared.

\section{Funding}

None.

\section{Ethical approval}

This study was approved by the corresponding the ethics committee is COMITÉ DE ÉTICA DE LA INVESTIGACIÓN CON MEDICAMENTOS DE EUSKADI (CEIm-E) (Code PI2020083) and conducted in accordance with the principles of the Declaration of Helsinki on research in humans.

\section{Author contributions}

LSF and LAR take responsibility for the manuscript as a whole. LSF, LAR, RZJ, RMV, RMO and PPE conceived and designed the study. AUE, PGH, PGJ, ETH, RMO and LAR enrolled patients, and collected and compiled data. SPF performed the statistical analysis. LSF, RZJ, LAR, RMO, ATM, PPE and RMV analysed and interpreted the data. LSF, RZJ and LAR wrote the manuscript. RMV, RMO, ATM, AUE and PPE commented on and revised the report. All authors read and approved the final manuscript.

\section{Supplementary materials}

Supplementary material associated with this article can be found, in the online version, at doi:10.1016/j.ijid.2021.11.023.

\section{References}

Altawalah H. Antibody response to natural SARS-CoV-2 infection or after COVID-19 vaccination. Vacciones (Basael) 2021;9(8):910. doi:10.3390/vacciones9080910.

Berenguer J, Ryan P, Rodríguez-Baño J, Jarrín I, Carratalà J, Pachón J, et al. COVID19@Spain Study Group. Characteristics and predictors of death among 4035 consecutively hospitalized patients with COVID-19 in Spain. Clin Microbiol Infect 2020;26(11):1525-36. doi:10.1016/j.cmi.2020.07.024.

Boumendil A, Angus DC, Guitonneau AL, Menn AM, Ginsburg C, Takun K, et al. ICECUB study group. Variability of intensive care admission decisions for the very elderly. PLoS One 2012;7(4):e34387. doi:10.1371/journal.pone.0034387.

Capelastegui A, Zalacain R, Bilbao A, Egurrola M, Ruiz LA, Quintana JM, et al. Pneumococcal pneumonia: differences according to blood culture results. BMC Pulm Med 2014;14(1):128. doi:10.1186/1471-2466-14-128.

Ciceri F, Castagna A, Rovere-Querini P, De Cobelli F, Ruggeri A, Galli L, et al. Early predictors of clinical outcomes of COVID-19 outbreak in Milan, Italy. Clin Immunol 2020;217:108509 108509. doi:10.1016/j.clim.2020.108509.

COVID-19 Treatment Guidelines Panel. Coronavirus disease 2019 (COVID-19) treatment guidelines. Bethesda, MD: National Institutes of Health; 2020 Available at https://www.covid19treatmentguidelines.nih.gov/ (accessed September 2020).

Dhama K, Khan S, Tiwari R, Sircar S, Bhat S, Malik YS, et al. Coronavirus disease 2019-COVID-19. Clin Microbiol Rev 2020;33(4):e00028-20 e00028-20. doi:10.1128/CMR.00028-20.

Documento técnico. Manejo clínico del COVID-19: tratamiento médico. Spanish Ministry of Health, Spanish Government; 19 March 2020 Available at: http:// www.aeemt.com/web/wpcontent/uploads/2020/03/4_6026300193912129107.pdf

Fine MJ, Smith MA, Carson CA, Mutha SS, Sankey SS, Weissfeld LA, et al. Prognosis and outcomes of patients with community-acquired pneumonia: a metaanalysis. JAMA 1996;275(2):134-41. doi:10.1001/jama.1996.03530260048030.
Fine MJ, Stone RA, Singer DE, Coley CM, Marrie TJ, Lave JR, et al. Processes and outcomes of care for patients with community-acquired pneumonia: results from the Pneumonia Patient Outcomes Research Team (PORT) Cohort Study. Arch Intern Med 1999;159(9):970-80. doi:10.1001/archinte.159.9.970.

Gallo Marin B, Aghagoli G, Lavine K, Yang L, Siff EJ, Chiang SS, et al. Predictors of COVID-19 severity: a literature review. Rev Med Virol 2021;31(1):1-10. doi:10.1002/rmv.2146.

Garnacho-Montero J, García-Cabrera E, Diaz-Martín A, Lepe-Jiménez JA, IraurgiArcarazo P, Jiménez-Alvarez R. Determinants of outcome in patients with bacteraemic pneumococcal pneumonia: importance of early adequate treatment. Scand J Infect Dis 2010;42(3):185-92. doi:10.3109/00365540903418522.

Gattarello S, Borgatta B, Solé-Violán J, Vallés J, Vidaur L, Zaragoza R, et al. Decrease in mortality in severe community-acquired pneumococcal pneumonia: impact of improving antibiotic strategies (2000-2013). Chest 2014;146(1):2231. doi:10.1378/chest.13-1531.

Grau I, Ardanuy C, Calatayud L, Schulze MH, Liñares J, Pallares R. Smoking and alcohol abuse are the most preventable risk factors for invasive pneumonia and other pneumococcal infections. Int J Infect Dis 2014;25:59-64. doi:10.1016/j.ijid.2013.12.013

Guo L, Wei D, Zhang X, Wu Y, Li Q, Zhou M, et al. Clinical features predicting mortality risk in patients with viral pneumonia: the MuLBSTA score. Front Microbiol 2019;10:2752. doi:10.3389/fmicb.2019.02752.

Hanada S, Iwata S, Kishi K, Morozumi M, Chiba N, Wajima T, et al. Host factors and biomarkers associated with poor outcomes in adults with invasive pneumococcal disease. PLoS One 2016;11(1):e0147877 e0147877. doi: $10.3109 / 00365540903418522$.

Ishiguro T, Kagiyama N, Uozumi R, Odashima K, Kurashima K, Morita S, et al. Risk factors for the severity and mortality of pneumococcal pneumonia: importance of premorbid patients' performance status. J Infect Chemother 2016;22(10):68591. doi:10.1016/j.jiac.2016.07.008.

Johansson N, Kalin M, Tiveljung-Lindell A, Giske CG, Hedlund J. Etiology of community-acquired pneumonia: increased microbiological yield with new diagnostic methods. Clin Infect Dis 2010;50(2):202-9. doi:10.1086/648678.

Levi M, Thachil J, Iba T, Levy JH. Coagulation abnormalities and thrombosis in patients with COVID-19. Lancet Haematol 2020;7(6):e438-40. doi:10.1016/S2352-3026(20)30145-9.

Levy MM, Fink MP, Marshall JC, Abraham E, Angus D, Cook D, et al. SCCM/ESICM/ACCP/ATS/SIS: $\quad$ SCCM/ESICM/ATS/SIS International Sepsis Definitions Conference. Crit Care Med 2003 2003;29(4):530-8. doi:10.1007/s00134-003-1662-X.

Lim WS, Van der Eerden MM, Laing R, Boersma WG, Karalus N, Town GI, et al. Defining community-acquired pneumonia severity on presentation to hospital: an international derivation and validation study. Thorax 2003;58(5):377-82. doi:10.1136/thorax.58.5.377.

Méndez R, Menéndez R, Amara-Elori I, Feced L, Piró A, Ramírez P, et al. Lymphopenic community-acquired pneumonia is associated with a dysregulated immune response and increased severity and mortality. J Infect 2019;78(6):42331. doi:10.1016/j.jinf.2019.04.006.

Menéndez R, Torres A, Aspa J, Capelastegui A, Prat C, Rodríguez de Castro F. Sociedad Española de Neumología y Cirugía Torácica: Communityacquired pneumonia. New guidelines of the Spanish Society of Pulmonary and Thoracic Surgery (SEPAR). Arch Bronconeumol 2010;46:543-58. doi:10.1016/j.arbres.2010.06.014.

Naucler P, Darenberg J, Morfeldt E, Ortqvist A. Henriques Normark B. Contribution of host, bacterial factors and antibiotic treatment to mortality in adult patients with bacteraemic pneumococcal pneumonia. Thorax 2013;68:571-9. doi: $10.1136 /$ thoraxjnl-2012-203106.

Nguyen Y, Corre F, Honsel V, Curac S, Zarrouk V, Fantin B, et al. Applicability of the CURB-65 pneumonia severity score for outpatient treatment of COVID-19. J Infect 2020;81(3):e96-8. doi:10.1016/j.jinf.2020.05.049.

Richardson S, Hirsch JS, Narasimhan M, Crawford JM, McGinn T, Davidson KW, et al. Presenting characteristics, comorbidities, and outcomes among 5700 patients hospitalized with COVID-19 in the New York City Area. JAMA 2020;323(20):2052-9. doi:10.1001/jama.2020.6775.

Roson B, Carratala J, Dorca J, Casanova A, Manresa F, Gudiol F. Etiology, reasons for hospitalization, risk classes, and outcomes of community-acquired pneumonia in patients hospitalized on the basis of conventional admission criteria. Clin Infect Dis 2001;33(2):158-65. doi:10.1086/321808.

Ruiz LA, España PP, Gómez A, Bilbao A, Jaca C, Arámburu A, et al. Age-related differences in management and outcomes in hospitalized healthy and wellfunctioning bacteremic pneumococcal pneumonia patients: a cohort study. BMC Geriatr 2017;17(1):130. doi:10.1186/s12877-017-0518-0.

Ruiz LA, Serrano L, España PP, Martinez-Indart L, Gomez A, Uranga A, et al. Factors influencing long-term survival after hospitalization with pneumococcal pneumonia. J Infect 2019;79(6):542-9. doi:10.1016/j.jinf.2019.10.024.

Said MA, Johnson HL, Nonyane BA, Deloria-Knoll M, O'Brien KL, et al. Estimating the burden of pneumococcal pneumonia among adults: a systematic review and meta-analysis of diagnostic techniques. PLoS One 2013;8(4):e60273. doi: 10.1371 /journal.pone.0060273.

Shariatzadeh MR, Huang JQ, Tyrrell GJ, Johnson MM, Marrie TJ. Bacteremic pneumococcal pneumonia: a prospective study in Edmonton and neighboring municipalities. Medicine 2005;84(3):147-61. doi:10.1097/01.md.0000164302.03972.d7.

Shi F, Xia L, Shan F, Wu D, Wei Y, Yuan H, et al. Large-scale screening of COVID-19 from community-acquired pneumonia using infection size-aware classification. Phys Med Biol 2021;66(6):06503 065031. doi:10.1088/1361-6560/abe838. 
Siddiqi HK, Mehra MR. COVID-19 illness in native and immunosuppressed states: a clinical-therapeutic staging proposal. J Heart Lung Transpl 2020;39(5):405. doi:10.1016/j.healun.2020.03.012.

Tian J, Xu Q Liu S, Mao L, Wang M, Hou X. Comparison of clinical characteristics between coronavirus disease 2019 pneumonia and community-acquired pneumonia. Curr Med Res Opin 2020;36(11):1747-52. doi:10.1080/03007995.2020.1830050.

Tratamientos disponibles sujetos a condiciones especiales de acceso para el manejo de la infección respiratoria por SARS-CoV-2. June 2020. Spanish Agency of Medicines and Medical Devices, Spanish Ministry of Health, Spanish Government. Available at: https://www.aemps.gob.es/ la-aemps/ultima-informacion-de-la-aemps-acerca-del-covid\%e2\%80\%9119/ tratamientos-disponibles-para-el-manejo-de-la-infeccion-respiratoria-por-sars-cov- 2 ?lang=en

Tratamientos disponibles para el manejo de la infección respiratoria por SARS-CoV2.18. March 2020. Spanish Agency of Medicines and Medical Devices, Spanish Ministry of Health, Spanish Government. Available at: https://www.aemps.gob. es/laAEMPS/docs/medicamentos-disponibles-SARS-CoV-2-19-3-2020.pdf

Van den Brand JM, Haagmans BL, van Riel D, Osterhaus AD, Kuiken T. The pathology and pathogenesis of experimental severe acute respiratory syndrome and influenza in animal models. J Comp Pathol 2014;151(1):83-112. doi:10.1016/j.jcpa.2014.01.004

Van der Poll T, Opal SM. Pathogenesis, treatment, and prevention of pneumococcal pneumonia. Lancet 2009;374(9700):1543-56. doi:10.1016/s0140-6736(09)61114-4.

Whitney CG, Farley MM, Hadler J, Harrison LH, Bennett NM, Lynfield R, et al. Active bacterial core surveillance of the Emerging Infections Program Network. Decline in invasive pneumococcal disease after the introduction of protein-polysaccharide conjugate vaccine. N Engl J Med 2003;348(18):1737-46. doi:10.1056/NEJMoa022823.
Wynants L, Van Calster B, Collins GS, Riley RD, Heinze G, et al. Prediction models for diagnosis and prognosis of COVID-19: systematic review ad critical appraisal. BMJ 2020;369:m1328. doi:10.1136/bmj.m1328.

Xu Z, Shi L, Wang Y, Zhang J, Huang L, Zhang C, et al. Pathological findings of COVID-19 associated with acute respiratory distress syndrome. Lancet Respir Med 2020;8(4):420-2. doi:10.1016/S2213-2600(20)30076-X.

Ye Q Wang B, Mao J. The pathogenesis and treatment of the 'cytokine storm' in COVID-19. J Infect 2020;80(6):607-13. doi:10.1016/j.jinf.2020.03.037.

Zhan N, Guo Y, Tian S, Huang B, Tian X, Zou J, et al. Clinical characteristics of COVID-19 complicated with pleural effusion. BMC Infect Dis 2021;21(1):176. doi:10.1186/s12879-021-05856-8.

Zhao D, Yao F, Wang L, Zheng L, Gao Y, Ye J, et al. A comparative study on the clinical features of COVID-19 pneumonia to other pneumonias. Clin Infect Dis 2020;71(15):756-61. doi:10.1093/cid/ciaa247.

Zheng Z, Peng F, Xu B, Zhao J, Liu H, Peng J, et al. Risk factors of critical \& mortal COVID-19 cases: a systematic literature review and meta-analysis. J Infect 2020;81(2):e16-25. doi:10.1016/j.jinf.2020.04.021.

Zhou F, Yu T, Du R, Fan G, Liu Y, Liu Z, et al. Clinical course and risk factors for mortality of adult in patients with COVID-19 in Wuhan, China: a retrospective cohort study. Lancet 2020a;395:1054-62. doi:10.1016/S0140-6736(20)30566-3.

Zhou Y, Guo S, He Y, Zuo Q, Lui D, Xiao M, et al. COVID-19 is distinct from SARS-CoV-2-negative community-acquired pneumonia. Front Cell Infect Microbiol 2020b;10:332. doi:10.3389/fcimb.2020.00322. 